\title{
Effects of intertrial interval and exteroceptive feedback duration on discriminative avoidance acquisition in the gerbil
}

\author{
PETER F. GALVANI and MICHAEL T. TWITTY \\ State University of New York, College at Brockport, Brockport, New York 14420
}

\begin{abstract}
A series of studies of shuttlebox-avoidance learning in the gerbil evaluated the efficacy of an exteroceptive feedback stimulus (FS). Experiment 1 assessed the relative effectiveness of a FS at 30- and 90-sec intertrial intervals (ITIs), and found that the FS and warning signal termination contingencies were additive sources of avoidance reinforcement; i.e., they produced "supernormal acquisition" at the short ITI, but not at the 90-sec ITI. The effectiveness of a FS at the 30-sec ITI was further explored in Experiments 2 and 3, in which FS duration was varied in delayed and trace avoidance conditioning, respectively. In both studies, a FS facilitated acquisition but FS duration was not a critical determinant of performance. These results were interpreted in terms of an expectancy account of the informational value of a FS, and the problem of experimentally distinguishing between cognitive and inhibition-of-fear accounts of avoidance learning was discussed.
\end{abstract}

There presently exists a considerable literature demonstrating the efficacy of an exteroceptive feedback stimulus (FS) on avoidance acquisition (Bolles \& Grossen, 1969, 1970; Bower, Starr, \& Lazarovitz, 1965; D'Amato, Fazarro, \& Etkin, 1968; Galvani, 1977; Keehn \& Nakkash, 1959). The typical finding has been that, in the absence of response-produced warning signal termination (WST), a responseproduced FS results in avoidance learning equivalent to that produced by the traditional WST contingency. Recent formulations of avoidance learning have proposed that the FS reinforces avoidance behavior because it functions as a safety signal (Bolles \& Grossen, 1970) or an inhibitor of fear (Morris, 1974; Weisman \& Litner, 1972). Regardless of the terms employed in conceptualizing the functional significance of feedback in controlling avoidance responses, there seems to be agreement that the efficacy of a FS can be quantified in terms of the duration of the shock-free interval that is contingent upon FS presentation (Bolles \& Grossen, 1970; Morris, 1974).

In the discriminative avoidance paradigm, the contingency between the avoidance response and FS insures that the FS consistently precedes the shock-

Supported by National Science Foundation Grant BO43556-001 awarded to the first author. This paper is based, in part, upon Michael Twitty's master's thesis, directed by P. F. Galvani. The comments and suggestions of David Burrows, Frederick Gravetter, George Pinckney, Linda Pinckney, and William Riddell are gratefully acknowledged. Reprints may be obtained from Peter F. Galvani, Department of Psychology, State University College at Brockport, Brockport, New York 14420. free intertrial interval (ITI). The only study assessing feed-back effects as a function of ITI ( $30 \mathrm{vs.} 90 \mathrm{sec}$ ), reported very poor performance (approximately $20 \%-25 \%$ avoidance) in a discrete-trial task at the short ITI, irrespective of whether WST or FS presentation were response-contingent (Bolles \& Grossen, 1970). Indeed, relative to the baseline provided by animals lacking both a WS and FS, evidence of learning was at best marginal. Moreover, Morris (1974, Experiment 3) has provided indirect evidence suggesting that a FS might not be an effective reinforcer at a short ITI. In a transfer-of-training test, he failed to obtain evidence of the development of inhibition to a FS at a 30-sec ITI, although conditioned inhibition to a FS was evidenced at an $180-\mathrm{sec}$ ITI. Taken together, these results raise serious questions about the effectiveness of feedback at a short ITI.

Accordingly, the purpose of Experiment 1 was to assess the efficacy of feedback at a short $(30-\mathrm{sec})$ compared to a long $(90-\mathrm{sec})$ ITI in the Mongolian gerbil. Previous research shows that this relatively new laboratory species acquires a shuttle-avoidance response at both of these intervals within a 100-trial session (Galvani, Twitty, \& Foster, 1975). The present design involved the independent manipulation of both the WST and FS contingencies at both ITIs. Based on previous evidence, as well as the theoretical view that the effectiveness of feedback depends upon the duration of the shock-free interval following the FS, one might assume that feedback should have little or no reinforceing effect at the short ITI, whereas at the 90-sec ITI it should be functionally equivalent to WST (cf. Bolles \& Grossen, 1969). 


\section{EXPERIMENT 1}

\section{Method}

Subjects. The subjects were 80 naive male gerbils, $3-4$ months old, obtained from Tumblebrook Farms, West Brookfield, Massachusetts. All subjects were maintained on ad-lib food and water, under a 12-h light-dark regimen, and were housed in groups of five.

Apparatus. The apparatus consisted of two Scientific Prototype Model AlOOS toggle-floor shuttleboxes $(13.5 \mathrm{~cm}$ high $\times 13 \mathrm{~cm}$ wide $\times 40 \mathrm{~cm}$ long) with Plexiglas sides and tops and aluminum ends. The shuttleboxes were divided in half by an aluminum insert with an arch-shaped cutout, $7 \mathrm{~cm}$ wide at the base $\times 9 \mathrm{~cm}$ in height at the center of the arch. The arch opening permitted passage from one side of the shuttlebox to the other. The shuttlebox floor consisted of stainless steel $.24-\mathrm{cm}$ grid bars, spaced $.95 \mathrm{~cm}$ apart, center-to-center. Each shuttlebox was enclosed in a ventilated, sound-attenuated chamber illuminated by a $7.5-\mathrm{W}$ incandescent lamp. A tonal $\mathrm{CS}(2,500 \mathrm{~Hz})$ was delivered through an 8-cm speaker, centered above each shuttlebox, via a BRSForinger AU-902 audio generator. The tone raised the ambient sound level ( $82 \mathrm{~dB}$ with ventilating fan on) about $3 \mathrm{~dB}$ on the C scale of a General Radio Company sound-level meter, Type 1561-A. The to-be-avoided aversive stimulus was a $.5-\mathrm{mA}$ (nominal) .5-sec scrambled electrical shock delivered to the grid floor of the shuttlebox by a Grason-Stadler Model GS 700 shock generator. The FS consisted of simultaneous offset of chamber illumination and venting fan (reducing ambient sound level to about $71 \mathrm{~dB}$ ). Programming and data recording equipment were located adjacent to the chambers housing the shuttleboxes.

Procedure. After a 10-min adaptation period in the shuttlebox with the chamber light and venting fan on, each gerbil received a 100 -trial acquisition session at either a 30 - or $90-\mathrm{sec}$ ITI. The WS-shock interval was $5 \mathrm{sec}$ on all trials, with onset of the .5-sec inescapable shock occurring simultaneously with WS of fset. Any shuttle response within $5 \mathrm{sec}$ of WS onset, but before shock delivery, was defined as a CR. All CRs precluded shock, regardless of other programmed consequences.

The 2 by 2 by 2 factorial design employed 10 animals per group. In addition to ITI, the other independent variables were the WST and FS contingencies. Following a CR, WST was either immediate (I) or delayed (D), and the exteroceptive FS was presented (F) or not presented (N). Thus, four groups, abbreviated as IF, IN, DF, and DN, were trained at each ITI. Duration of both FS presentation and WST delay following a CR was $10 \mathrm{sec}$, measured from the initial CR occurrence on a given trial. For all analyses and comparisons reported as reliable in the present experiment, $a=.05$.

\section{Results}

The CR frequency data of Experiment 1 are presented in Figure 1 over blocks of training trials as a function of the programmed consequences of a CR. The 30- and 90-sec groups have been plotted separately in the upper and lower panels of Figure 1, respectively. Analysis of these data revealed that the $90-\mathrm{sec}$ group was superior in acquisition performance to the 30 -sec animals $[F(1,72)=29.82]$, thus replicating Galvani et al. (1975). In order to further simplify presentation of the results, separate mixed analyses of variance (ANOVA) were performed on the 30- and 90-sec groups. Each ANOVA involved the WST contingency (I vs. D) and FS contingency ( $F$ vs. $N$ ) as between-subjects variables, and repeated measures on the trial blocks variable. Separate

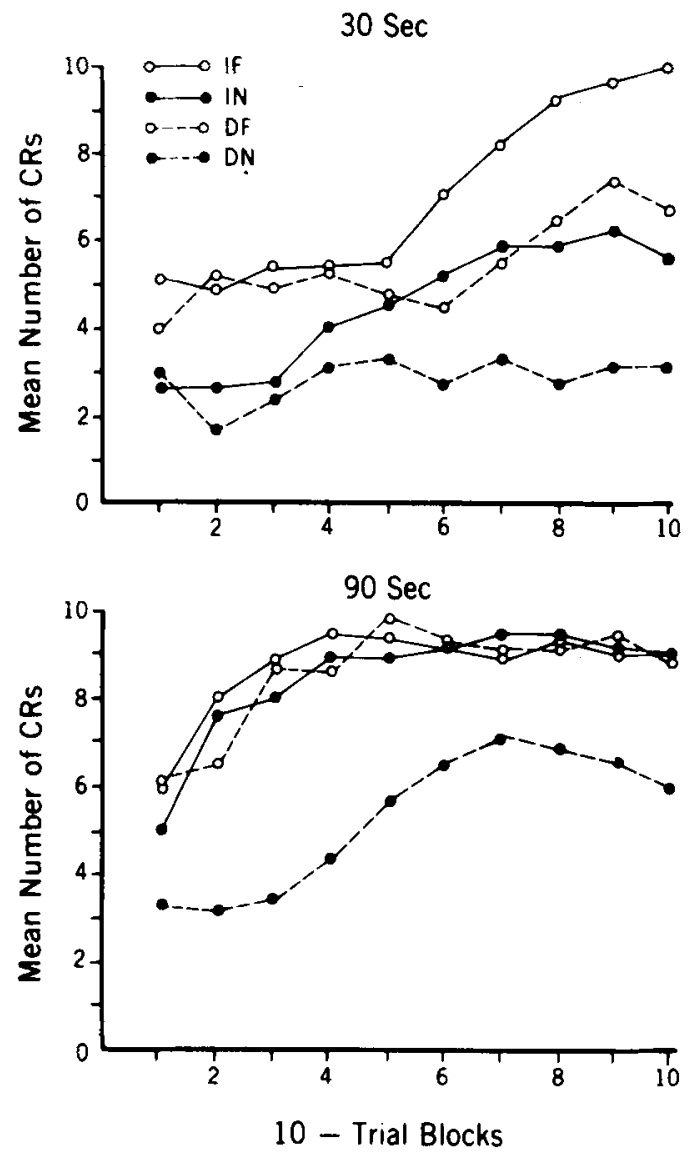

Figure 1. Mean number of avoidance CRs in 10-trial blocks as a function of whether the CR produced immediate (I) or delayed (D) WS termination, and whether or not (F or N) it produced feedback. The separate panels plot groups as a function of ITI duration ( 30 or 90 sec).

ANOVAs were performed on the data of Trial Blocks 1-5 and 6-10.

The ANOVA of the 30-sec-ITI CR frequency data on Blocks $1-5$ resulted in only one significant effect - feedback $[F(1,36)=4.75]$; no other main effects or interactions were reliable. Over Blocks 6-10, however, all main effects were significant: WST $[F(1,36)$ $=7.46]$, feedback $[F(1,36)=8.8]$, and trial blocks $[F(4,144)=7.42]$. The only significant interaction involved feedback and trial blocks $[F(4,144)=4.35]$. This interaction presumably resulted from the feedback groups' diverging from the nonfeedback groups later in training as evident from inspection of Figure 1. No reliable divergence occurred, however, between immediate and delayed WST groups.

These analyses provide statistical support for the following description of the $30-\mathrm{sec}$ ITI results. During approximately the first 50 trials of acquisition, the feedback groups were superior to the nonfeedback groups, but this superiority did not vary systematically over trial blocks. In the second half of the session, however, both the WST and FS contingen- 
cies provided separate, additive (interaction $\mathrm{F}<1$ ) sources of reinforcement for the CR. Indeed, if either contingency were operative alone (Groups IN and DF), performance was about midway between that resulting when both contingencies were effective (Group IF) and when neither contingency was in effect (Group DN). These results point to the conclusion that, with sufficient training at a short ITI, WST and presentation of an exteroceptive FS function as two distinguishable sources of reinforcement for avoidance learning in the gerbil.

At the 90 -sec ITI, a rather different pattern of results obtained. Obviously, avoidance learning was more rapid at the long ITI. The groups that experienced both contingencies, or either a FS with delayed WST or immediate WST alone (Groups IF, DF, and IN, respectively), performed equally well, while Group DN was clearly inferior to all three. The ANOVA on Blocks $1-5$ of these data indicated that all main effects were reliable: WST $[F(1,36)$ $=21.66]$, feedback $[F(1,36)=27.62]$, and trial blocks $[F(4,144)=22.31]$. In contrast to the analyses of the 30-sec ITI data, however, the present analysis revealed. a significant WST by Feedback interaction $[F(1,36)=14.95]$. Thus, the WST and FS contingencies did not operate as separable, additive sources of reinforcement at the $90-\mathrm{sec}$ ITI. Finally, WST interacted with trial blocks $[F(4,144)$ $=2.94]$, but no other interaction was significant (Fs $<1$ ). Over Trial Blocks 6-10, the only reliable effects were: WST $[F(1,36)=5.07]$, feedback $[F(1,36)=4.57]$, and their interaction $[F(1,36)=$ 7.74]. Thus, the results of the ANOVAs on the first and second halves of acquisition were consistent, except for the effects of trial blocks. The failure of trial blocks to be significant later in training was presumably due to all groups' attaining asymptotic performance levels by Blocks 6-10.

\section{Discussion}

These results do not support the view that a FS is ineffective in facilitating avoidance acquisition at a short ITI. Two aspects of the present data are contrary to such an assertion. First, introduction of an exteroceptive FS was effective at the short as well as the long ITI in reducing the performance decrement found with delayed WST, producing comparable avoidance performance to the traditional procedure of immediate WST without a FS. This evidence shows that a FS can take over the role of immediate WST, despite the fact that the time interval following the FS is brief. Second, at the 30-sec ITI, Group IF was superior to IN (a result referred to as "supernormal acquisition" by Bolles \& Grossen, 1969), demonstrating that the combination of a FS and WST can facilitate avoidance performance relative to that obtained with WST alone. Perhaps this latter outcome implies that the FS was more effective at the 30-sec ITI than at the 90-sec interval, where supernormal acquisition was not found. On the other hand, the failure to obtain supernormal acquisition at the longer ITI might simply be due to a ceiling effect.

The roughly equivalent performance levels of Groups DF and IN, noted at both ITIs (though only later in training at the $30-\mathrm{sec}$ ITI), is consistent with a general rule that the combination of delayed WST and a FS is functionally equivalent to immediate WST in reinforcing avoidance (Bolles \& Grossen, 1969; Bower et al., 1965; Cicala \& Owen, 1976; D'Amato et al., 1968; Galvani, 1977; Keehn \& Nakkash, 1959). The supernormal acquisition effect obtained at the 30-sec ITI, however, has seldom been reported. At the time we collected these data, in fact, there was only one published demonstration of summation of the WST and FS contingencies (Bolles \& Grossen, 1969, Experiment 3), and this experiment employed a long ITI; on the other hand, there were numerous cases in which the IF and IN treatments resulted in similar performance (Bolles \& Grossen, 1969, Experiments 4 \& 5, 1970; Bower et al., 1965, Experiment 3; Cicala \& Owen, 1976, Experiment 1; Galvani, 1977; Katzev \& Hendersen, 1971, Experiment 1). More recently, however, supernormal acquisition has been reported at a 35-sec ITI (Cicala \& Owen, 1976, Experiments 2 \& 3; Cicala, Owen, \& Hill, 1976), demonstrating that the present finding has some generality. In those instances in which supernormal acquisition has not been found, including the present results at the 90 -sec ITI, avoidance acquisition has been relatively fast, suggesting that ceiling effects have militated against supernormal acquisition. This view is further supported by the observation that successful demonstrations of reinforcement summation have occurred only when animals experiencing immediate WST alone have performed at an intermediate level (e.g., asymptote around $60 \%$ avoidances in present study and in Bolles \& Grossen, 1969, Experiment 3). Such speculations suggest the conclusion that, at the empirical level, WST and FS presentation are separate, additive sources of avoidance reinforcement, and supernormal acquisition is likely to be found unless precluded by ceiling effects.

Finally, the superiority of the FS groups early in training at the 30 -sec ITI poses a problem. Perhaps the effect is attributable to unconditioned reinforcing properties of the FS, but then it still remains unclear why no progressive $C R$ strengthening occurred over the first five trial blocks.

\section{EXPERIMENT 2}

The purpose of the present study was to obtain further information concerning the facilitative effect of a FS on shuttle-avoidance performance at a short 
ITI. It seems reasonable to assume that the FS functioned primarily as a salient signal that shock had been avoided in Experiment 1. In these terms, the degree of response-produced stimulus change would be of paramount importance but a FS parameter such as temporal duration should be of no particular consequence. If a brief FS is sufficiently salient to facilitate performance, extending FS duration should not provide more salient cues to the animal. Accordingly, the design varied duration of the FS presented ( 2 to $10 \mathrm{sec}$ ), and compared the resultant performance to that produced by the traditional avoidance training procedure without FS presentation. Also, it is noteworthy that, if the FS of Experiment 1 did have unconditioned reinforcing effects, varying the duration of exposure to the FS might result in performance differences between groups.

\section{Method}

Subjects. Sixty-four naive male gerbils, 3-4 months old, served as subjects. The supplier and maintenance conditions were as in Experiment 1.

Apparatus. The same apparatus, programming, and datarecording equipment were used as previously. All experimental parameters were exactly as in Experiment 1, except that the ITI was $30 \mathrm{sec}$ for all subjects.

Procedure. The adaptation period and acquisition session were conducted as in Experiment 1. The design consisted of four groups $(n=16$ each), all of which received immediate WST and shock omission following a CR. For gerbils trained under IF2, IF5, and IF10 conditions, a CR resulted in immediate FS presentation of $2-, 5-$, and 10-sec duration, respectively. IN-trained gerbils received only immediate WST following a $C R$. For all animals, if no CR occurred during the 5-sec WS, shock presentation was coincident with WST.

\section{Results}

The mean number of CRs in acquisition are depicted in Figure 2 for each group in 10-trial blocks.

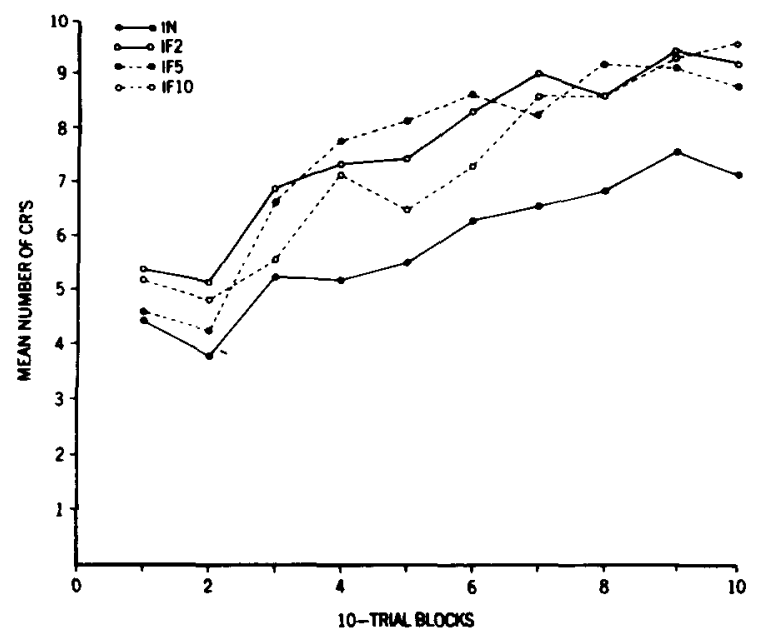

Figure 2. Mean number of avoidance CRs in 10-trial blocks as a function of programmed consequences of a $\mathbf{C R}$. [For separate groups, a CR produced no feedback (N) or either a 2-, 5-, or 10 -sec feedback (F) stimulus. All groups received immediate (I) WS termination.]

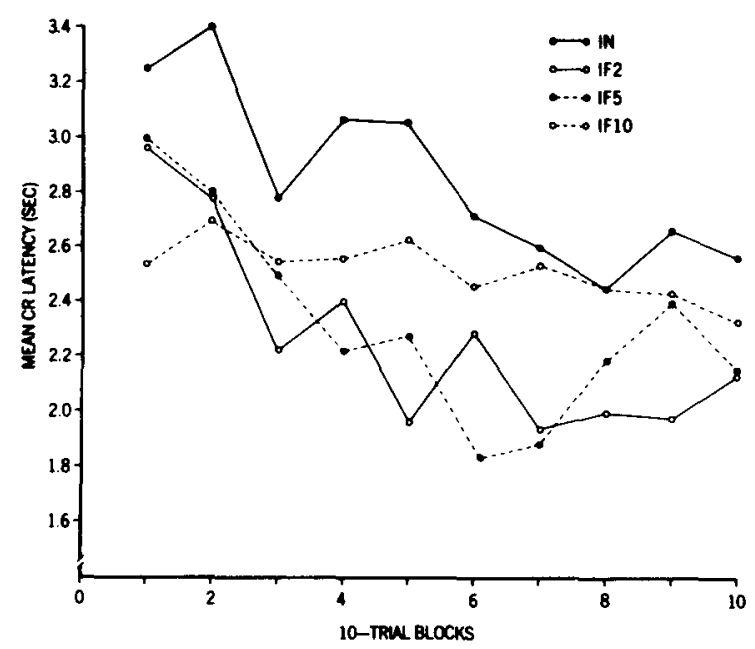

Figure 3. Mean CR latency in 10-trial blocks as a function of programmed consequences of a $\mathrm{CR}$.

Inspection of Figure 2 suggests that superior avoidance performance was obtained by each of the three FS groups relative to Group IN. Separate analyses were performed on the first and second halves of acquisition, as previously done in Experiment 1. Over Blocks 1-5, a nonsignificant effect of FS duration was observed $(F<1)$. The effect of trial blocks, however, was significant $[F(4,240)=14.77]$, but not the FS Duration by Trial Blocks interaction $[F(12,240)$ $=1.12 \mathrm{l}$. The ANOVA performed on Blocks 6-10 yielded a reliable main effect for FS duration $[F(3,60)=3.96]$ and trial blocks $[F(4,240)=5.14]$, but their interaction was not significant $(F<1)$. A Newman-Keuls post hoc analysis revealed that Group IN differed significantly from Groups IF2, IF5, and IF10. None of the FS groups differed reliably from each other.

Response latencies were also employed as a means of assessing avoidance performance. For each animal, the median latency score for CRs in a given 10-trial block was used as the individual subject's datum in computing group mean latencies per trial block. Figure 3 presents the group means, and it shows that throughout the 100-trial acquisition session, Group IN maintained longer mean CR latencies than the FS groups. Separate ANOVAs were performed on these latency scores for Blocks 1-5 and 6-10. The ANOVA on the first half of acquisition showed that the effect of trial blocks $[F(4,240)=$ 3.03] was significant, but the effects of FS duration $[F(3,60)=2.45]$ and the interaction $(F<1)$ were not reliable. During the second half of acquisition only the effect of FS duration $[F(3,60)=3.9]$ was significant. A follow-up Newman-Keuls analysis on the data of Blocks 6-10 indicated that reliable treatment differences emerged only between Group IN and Groups IF2 and IF5. While mean CR latencies in general were apparently shorter for the three FS 
groups than Group IN, the effect was significant in only two of the three comparisons.

\section{Discussion}

These results show that, although the presence of a FS facilitated acquisition, FS duration was not a determinant of avoidance performance. These data are in agreement with those of Experiment 1 in showing supernormal acquisition at a 30-sec ITI. A strictly cognitive interpretation, such as the information hypothesis proposed by Bolles and Grossen (1969), could readily be applied to the Experiment 2 results. According to such a view, response-produced feedback provides information to the subject, i.e., signals successful shock avoidance. Presumably, the 2-sec FS was just as predictive of successful shock avoidance as the 5- or 10-sec FSs, and therefore FS duration had no effect. Since simultaneous WST and FS onset apparently provided a more salient stimulus change than WST alone, the greater degree of stimulus change contingent on the CR in FS groups relative to Group IN facilitated the discrimination of the avoidance contingency.

One aspect of the present study of particular interest was the finding that both $\mathrm{CR}$ measures, frequency and latency, provided comparable results. Bolles, Moot, and Nelson (1976) recently noted that CR latencies are not typically reported as a measure in avoidance learning experiments. Moreover, Bolles et al. reported $C R$ latencies were relatively invariant over trials. The present results clearly contradict their findings. Response latencies not only varied significantly later in training as a function of whether or not a FS was CR-contingent, but mean CR latencies also decreased reliably over Trial Blocks 1-5. Finally, no evidence that the FS had unconditioned reinforcing effects was obtained in the present study.

\section{EXPERIMENT 3}

Although FS duration had no discernible effect over the 2-10 sec range explored in Experiment 2, the possibility of a performance ceiling cannot be ruled out, especially since avoidance acquisition in Group IN in the second study was superior to that found at the 30-sec ITI in Experiment 1. Incidentally, the Group IN performance of Experiment 2 is more representative of the performance obtained under these conditions in previous studies in the Brockport laboraties (e.g., Galvani, 1974; Galvani et al., 1975). Presumably these group differences between studies is simply due to sampling error. In order to provide further assessment of the effects of FS duration on avoidance acquisition, Experiment 3 replicated the previous study, employing trace conditioning rather than the delayed procedure of Experiments 1 and 2.
Since the trace procedure has been shown to retard acquisition relative to delayed conditioning (e.g., Black, 1963), its use should minimize the ceiling effect problem. Also, since WST is not possible in trace avoidance conditioning, the only exteroceptive stimulus change contingent on a $C R$ would be the FS. Accordingly, the trace procedure should aug ment the degree of control exerted by the responsecontingent FS, thereby providing a more sensitive test of FS duration effects than the delayed conditioning procedure.

\section{Method}

Subjects. The subjects were 48 naive male gerbils, $3-4$ months old, obtained from the same supplier and maintained as in Experiments 1 and 2.

Apparatus. The same apparatus was employed as in previous experiments. All parameters and conditions of the experiment were the same as Experiment 2, except that the WS was a fixed. duration, l-sec tone.

Procedure. Adaptation and acquisition were run as in Experiment 2. Four groups $(n=12$ each) received trace conditioning at a 5-sec WS onset-shock interval, and the CR-contingent FS was of $0-, 2-, 5-$, or 10-sec duration in Groups TN, TF2, TF5, and TF10, respectively. The WS was not CR-terminated. All CRs precluded shock, but if no CR occurred, then the 0.5 -sec shock was presented $4 \mathrm{sec}$ after WS offset.

\section{Results}

The CR frequency data are shown in Figure 4. Although these acquisition curves reveal inferior performance compared to Figure 2, it is still clear that there was substantial learning under trace conditioning, except in Group TN. A 4 (FS duration) by 5 (Trial Blocks 1-5) ANOVA of these data indicated that trial blocks $[F(4,176)=4.38]$ was significant, but not FS duration $[F(3,44)=1.7]$ or the interaction $[F(12,176)=1.39]$. The ANOVA on Blocks

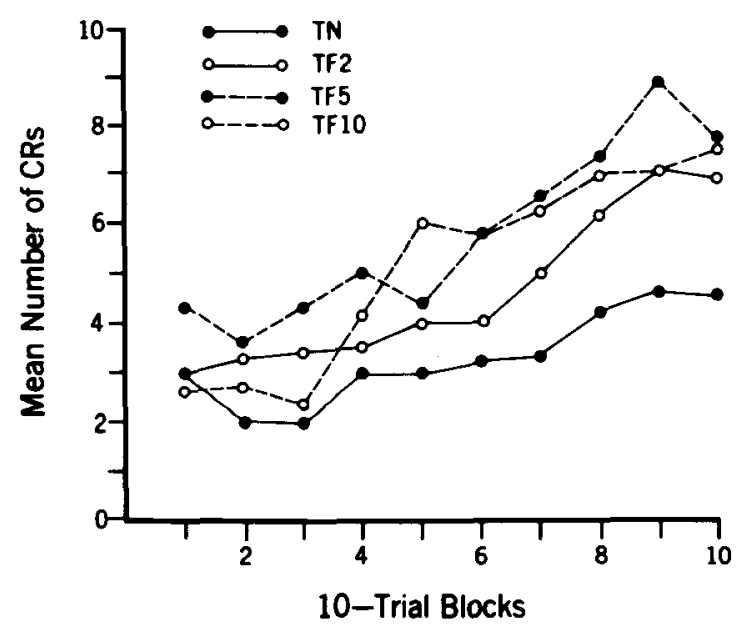

Figure 4. Mean number of CRs in 10-trial blocks as a function of feedback duration $[10,5,2$, or 0 (N) sec]. All groups received trace $(T)$ conditioning. 
6-10 revealed that both FS duration $[\mathrm{F}(3,44)=3.48]$ and trial blocks $[F(4,176)=12.97]$ were significant, but not their interaction $(F<1)$. A follow-up test of FS duration employed a Newman-Keuls analysis, collapsed across Blocks 6-10. Group TN differed from both TF5 and TF10, but TN and TF2 did not differ significantly. From an inspection of Figure 4, however, we believe it reasonable to assume that by the end of the 100-trial session, the three FS groups were quite comparable, and superior to TN. Admittedly, Group TF2 displayed a more gradual rate of improvement compared to TF5 and TF10. Group TN, on the other hand, evidences little, if any, learning. To evaluate this latter possibility, follow-up tests comparing $\mathrm{CR}$ frequency in Blocks 1 and 10 were performed, and the TN group did not improve reliably $[F(1,11)=2.11]$. All $F S$ groups produced clear evidence of learning [all $F s(1,11) \geqslant 10.35$ ]. Thus, we conclude that the TF2 subjects did demonstrate avoidance learning, and all feedback groups attained similar terminal performance levels.

Once again, CR latencies were recorded and means of medians computed as in Experiment 2. These results appear in Figure 5 . Here it can be seen that latencies were quite variable early in training, but by Blocks 7-10 between-group differences had clearly emerged. An ANOVA of the latency data over Blocks $1-5$ indicated no reliable effects of FS duration $(F<1)$, or trial blocks $[F(4,176)=2.1]$, and no interaction $(F<1)$. The analysis of the $C R$ latency data of Blocks 6-10, however, demonstrated a reliable effect of FS duration $[F(3,44)=4.91]$, but trial blocks $[F(4,176)=2.14]$ and the interaction were nonsignificant. Although the trial-blocks variable was not reliable $(p<.10)$ in either half of acquisition, the effect of this variable was reliable over the entire session $[F(9,396)=2.34]$.

\section{Discussion}

The results of Experiment 3 closely paralleled those of Experiment 2. In terms of both response measures, frequency and latency, the presence of a FS facilitated acquisition, but FS duration over the 2-10-sec range did not appear to be a significant determinant of responding. In fact, in Experiment 3, employing trace conditioning, it can be concluded that avoidance learning failed to occur at all unless the CR produced a FS. This outcome suggests that strong control was exerted by the exteroceptive FS and that, in its absence, other possible sources of feedback, exteroceptive or interoceptive, did not provide adequate reinforcement.

Perhaps it should be stressed that in both Experiments 2 and 3 , the $C R$ latency measure provided a sensitive index of presence vs. absence of a FS, as well as evidence of improved performance over trial blocks. These results are at variance with the findings of Bolles et al. (1976), and show that avoid-

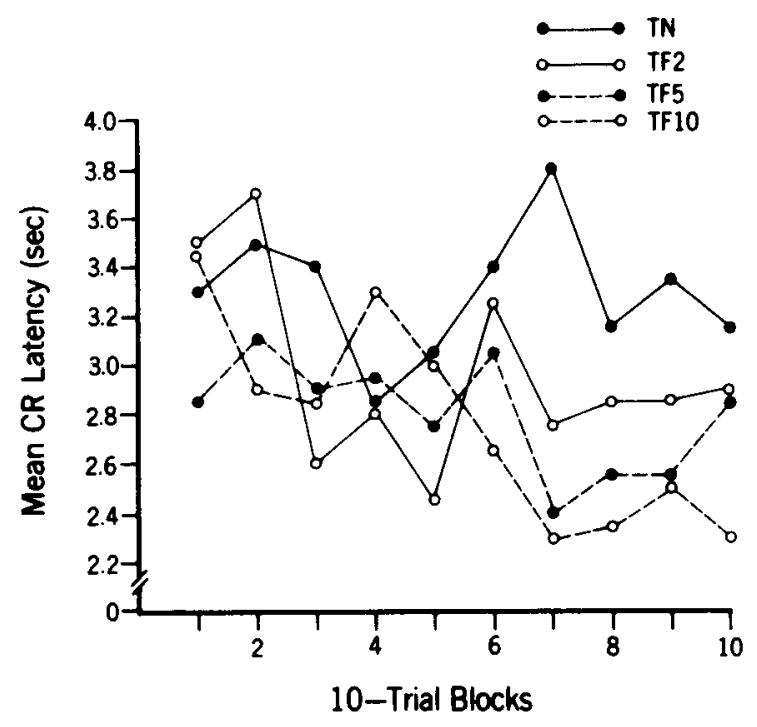

Figure 5. Mean CR latency in 10-trial blocks as a function of feedback duration in trace conditioning.

ance responses do not remain stationary in time as claimed by those authors.

Theoretically, the outcome of Experiment 3 can be readily viewed in informational terms. Thus, the FS provided reinforcement that might be conceptualized as a cue which reliably predicted successful shock avoidance. All FS groups experienced identical CRcontingent stimulus change, for the first $2 \mathrm{sec}$ post$\mathrm{CR}$ at least, and the duration of the signal beyond that point had little impact on avoidance learning.

\section{GENERAL DISCUSSION}

The present series of studies leaves no doubt that a CR-contingent exteroceptive FS can effectively reinforce shuttle avoidance learning in the Mongolian gerbil. The FS is effective, moreover, when WST is eliminated by the delayed offset procedure or by the use of trace avoidance conditioning. In addition, and perhaps more significantly, a FS facilitates avoidance acquisition even in the presence of immediate WST the supernormal acquisition effect. We agree with Cicala and Owen (1976) that the WST and FS presentation contingencies are separable, additive sources of avoidance reinforcement. The frequently reported failures to obtain supernormal acquisition, we suspect, are artifactual, i.e., due to ceiling effects on performance. Also, the present studies, along with other recent evidence, show convincingly that the FS facilitates acquisition even at short (30-35 sec) ITIs.

Cicala and Owen (1976) have claimed that the finding that the combination of WST and a FS is a more effective reinforcer than WST alone provides convincing evidence that WST and the FS serve different functions. We do not agree. Although super- 
normal acquisition indicates separable reinforcing functions of WST and FS at the empirical level, this does not constitute evidence of different reinforcing functions at the theoretical level. In other words, these two variables might affect the same underlying process and simply have additive effects.

The robust effect of the feedback contingency at the short ITI should not necessarily be viewed as incompatible with Weisman and Litner's (1971) inhibition-of-fear interpretation of avoidance acquisition. Clearly, ITI had a highly significant effect on avoidance acquisition in Experiment 1, perhaps attributable to greater conditioning of inhibition to feedback at the 90-sec ITI. Nevertheless, Morris' (1974) contention that FSs will have little or no effect upon performance unless they are fear inhibitors and that they will only become inhibitory if associated with long ITIs is seriously questioned by the present results. If a FS must become a conditioned inhibitor of fear to be effective, then the lack of independent evidence that a stimulus can become an inhibitor at such a short ITI creates problems (Morris, 1974; Moscovitch \& Lo Lordo, 1968; Weisman \& Litner, 1971). Either we must assume that the FS did become a conditioned inhibitor at a 30-sec ITI in the present studies or else that some other mechanism(s) must be proposed to explain the efficacy of the FS at the short ITI. A cognitive (informational) hypothesis of FS effects need not be constrained by the ITI variable (Bolles \& Grossen, 1969; D'Amato et al., 1968), though some cognitive views are so committed (e.g., safety-signal hypothesis, Bolles \& Grossen, 1970).

Interestingly enough, there are reasonable grounds for the inference that the FS gradually became a conditioned reinforcer at the 30-sec ITI in Experiment 1. Bolles (1972) has suggested that it requires about 40-60 trials in a typical shuttlebox situation for a FS to become a safety signal (or conditioned fear inhibitor), and it was at this approximate stage of training that Figure 1 shows that the feedback groups began to diverge from the nonfeedback groups (cf. Morris, 1975), and this divergence was found to be statistically reliable. Moreover, in both Experiments 2 and 3, the feedback groups were significantly superior to the nonfeedback groups only during the second half of acquisition in terms of both $\mathrm{CR}$ frequency and latency measures. Thus, these results also point to the conclusion that whatever mechanism(s) mediates the FS effect, a FS only gradually acquires the functional property of effectively reinforcing avoidance behavior.

While the present authors favor a cognitive interpretation of the effects of feedback, we are not convinced that revisions of two-factor theory (Cicala \& Owen, 1976; Morris, 1975; Weisman \& Litner, 1972) are not viable. Indeed, we believe that, as presently formulated, the cognitive and inhibition-of-fear hypotheses are not readily distinguishable. To illustrate, an informational interpretation such as that offered by Bolles and Grossen (1969) bears a striking similarity to the inhibition-of-fear conditioned reinforcement interpretation of feedback's functional significance. According to both views, empirically, a FS acquires its reinforcing value due to a negative correlation with shock. While the inhibition-of-fear hypothesis assumes that the FS acquires fearinhibiting properties over the course of avoidance trials to mediate performance, the information hypothesis assumes that the FS "tells" the subject it has "changed the situation" to the extent that the subject has previously acquired the expectancy that the FS predicts a nonshock interval. The behavioral prediction is singular; FS presentation facilitates avoidance performance only after the FS has acquired some "value." Perhaps it is the case that these formulations will be more clearly articulated only as more data are accumulated relative to the variables that determine the effectiveness (value) of exteroceptive feedback in avoidance learning.

\section{REFERENCES}

BLACK. A. H. The effect of CS-US interval on avoidance conditioning in the rat. Canadian Journal of Psychology, 1963, 17, 174-182.

Botles, R. C. The avoidance learning problem. In $G$. $H$. Bower (Ed.), The psychology of learning and motivation (Vol. 6). New York: Academic Press, 1972. Pp. 97-146.

Bolles, R. C., \& Grossen, N. E. Effects of an informational stimulus on the acquisition of avoidance behavior in rats. Journal of Comparative and Physiological Psychology, 1969. 68. 90.99 .

Bolles, R. C., \& Grossen, N. E. Function of the CS in shuttlebox avoidance learning by rats. Journal of Comparative and Physiological Psychology. 1970, 70. 165-169.

Bolles, R. C., Moot, S. A., \& Nelson, K. Note on the invariance of response latency in shuttlebox avoidance learning. Learning and Motivation, 1976, 7. 108-116.

Bower, G., Starr, R., \& Lazarovitz, L. Amount of response-produced change in the $\mathrm{CS}$ and avoidance learning. Journal of Comparative and Physiological Psychology, 1965. 59, 13-17.

Cicala. G. A., \& Owen. J. W. Warning signal termination and a feedback signal may not serve the same function. Learning and Motivation, 1976, 7, 356-367.

Cicala, G. A., Owen, J. W., \& Hill, D. Successful shuttle avoidance learning with high-intensity USs is sustained if a feedback signal accompanies warning-signal termination. Bulletin of the Psychonomic Society, 1976, 7, 533-535.

D'Amato, M. R., Fazzaro, J., \& Etkin, M. Anticipatory responding and avoidance discrimination as factors in avoidance learning. Journal of Experimental Psychology, 1968, 77, 41-47.

Galvani, P. F. Role of unconditioned stimulus escape and avoidance in discriminative avoidance learning in the gerbil (Meriones unguiculatus). Journal of Comparative and Physiological Psychology, 1974, 86, 846-852.

Galvani, P. F. Effects of feedback on discriminative avoidance acquisition and extinction in the gerbil (Meriones unguiculatus). Bulletin of the Psychonomic Society. 1977, 10. 304-306.

Galvani, P. F.. Twitty, M. T., \& Foster, K. M. Intermittent reinforcement and intertrial interval effects on shuttlebox 
avoidance in gerbil. Bulletin of the Psychonomic Society, $1975,5,225-227$.

Katzev, R. D.. \& Hendersen, R. W. Effects of exteroceptive feedback stimuli on extinguishing avoidance responses in Fischer344 rats. Journal of Comparative and Physiological Psychology, 1971, 74, 66.74.

KeEHN, D. J., \& NaKKash, S. Effect of a signal contingent upon an avoidance response. Nature (London), 1959, 184, $566-568$.

Morris, R. G. M. Pavlovian conditioned inhibition of fear during shuttlebox avoid ance behavior. Learning and Motivation, 1974, 5, 424-447.

Morris, R. G. M. Preconditioning of reinforcing properties to an exteroceptive feedback stimulus. Leaming and Motivation, 1975, 6. 289-298.
Moscovitch, A., \& LoLordo, V. M. Role of safety in the Pavlovian backward fear conditioning procedure. Joumal of Comparative and Physiological Psychology, 1968, 66. 673-678.

WEISMan, R. G., \& LITNER, J. S. Role of intertrial interval in Pavlovian differential conditioning of fear in rats. Journal of Comparative and Physiological Psychology, 1971, 74, 211.218.

Weisman, R. G.. \& Litner, J. S. The role of Pavlovian events in avoidance learning. In $R$. A. Boakes \& $M$. $S$. Halliday (Eds.), Inhibition and learning. New York: Academic Press, 1972. Pp. 253-270.

(Received for publication July 26, 1977; revision accepted November 5, 1977.) 